\title{
Lepton Portal dark matter
}

\author{
Yang $\mathrm{Bai}^{a}$ and Joshua Berger ${ }^{b}$ \\ ${ }^{a}$ Department of Physics, University of Wisconsin, \\ Madison, WI 53706, U.S.A. \\ ${ }^{b} S L A C$ National Accelerator Laboratory, \\ 2575 Sand Hill Road, Menlo Park, CA 94025, U.S.A.
}

E-mail: yangbai@physics.wisc.edu, jberger@slac.stanford.edu

ABSTRACT: We study a class of simplified dark matter models in which dark matter couples directly with a mediator and a charged lepton. This class of Lepton Portal dark matter models has very rich phenomenology: it has loop generated dark matter electromagnetic moments that generate a direct detection signal; it contributes to indirect detection in the cosmic positron flux via dark matter annihilation; it provides a signature of the sameflavor, opposite-sign dilepton plus missing transverse energy at colliders. We determine the current experimental constraints on the model parameter space for Dirac fermion, Majorana fermion and complex scalar dark matter cases of the Lepton Portal framework. We also perform a collider study for the $14 \mathrm{TeV}$ LHC reach with 100 inverse femtobarns for dark matter parameter space. For the complex scalar dark matter case, the LHC provides a very stringent constraint and its reach can be interpreted as corresponding to a limit as strong as two tenths of a zeptobarn on the dark matter-nucleon scattering cross section for dark matter masses up to $500 \mathrm{GeV}$. We also demonstrate that one can improve the current collider searches by using a Breit-Wigner like formula to fit the dilepton MT2 tail of the dominant diboson background.

Keywords: Beyond Standard Model, Cosmology of Theories beyond the SM

ARXiv EPRINT: 1402.6696 


\section{Contents}

1 Introduction 1

2 A simplified dark matter model: the Lepton Portal 3

3 Relic abundance $\quad 4$

4 Dark matter direct detection 5

5 Dark matter indirect detection $\quad 9$

6 Collider constraints and searches $\quad 11$

$\begin{array}{lll}7 & \text { Discussion and conclusions } & 14\end{array}$

$\begin{array}{ll}\text { A Non-relativistic correspondence of photon couplings } & 15\end{array}$

$\begin{array}{lr}\text { B Lepton } g-2 & 17\end{array}$

\section{Introduction}

The search for thermal relic Weakly Interacting Massive Particle (WIMP) dark matter has a long history, particularly within models of weak-scale supersymmetry (SUSY) [1, 2]. Such models can furnish both signatures of new physics at the $\mathrm{TeV}$ scale and a viable candidate for dark matter (DM). Collider, direct detection and indirect detection searches for Minimal Supersymmetric Standard Model (MSSM) dark matter particles have limited the vanilla parameter space $[3,4]$ and weaken the strong tie between WIMP dark matter and the SUSY framework. Outside of weak-scale SUSY models, there is no specific reason for dark matter to have mass near $100 \mathrm{GeV}$. Fortunately, the "WIMP miracle" provides guidance for the plausible region of dark matter mass and interaction strength [5]. Since even the discovery of dark matter from multiple experimental probes is unlikely to immediately tell us the underlying framework, in this paper we concentrate on a class of simplified dark matter models, which serves as a phenomenological bridge between experiments and a deep underlying theory.

There have been a number of recent studies of simplified dark matter models with the emphasis on the complimentarity from different experimental searches [6-13]. Most of those studies have concentrated on dark matter interactions with the quarks of the Standard Model (SM), which leads to a new framework for interpretation of LHC and direct detection searches in terms of dark matter properties. For instance, in ref. [8] the signature of two jets plus missing transverse energy has been studied within the context 
of Quark Portal dark matter models, which is a class of simplified models in which dark matter particles and mediators interact with a single quark. In this paper, following our previous study in ref. [8], we concentrate on the lepton sector and study a class of Lepton Portal dark matter models. In these models, there are two new particles in the dark matter sector with the lightest one being the dark matter candidate, which must be a singlet under electromagnetism and color. The other particle plays the role of mediator and connects the dark matter particle to the leptons. In the models we consider, to conserve the SM gauge symmetry, the mediator particle should be charged under the electroweak symmetry. For the dark matter interactions to be renormalizable, the mediator must have the same quantum numbers as the left-handed lepton weak doublet or the right-handed charged leptons. In our study, we consider only the latter case for simplicity. It is worth noting that this structure differs from models that admit $s$-channel DM annihilation. In those models, the mediator can be a SM gauge singlet, as in $Z^{\prime}$-mediated leptophilic models for example [14-16].

Compared to Quark Portal dark matter models, Lepton Portal dark matter models have totally different phenomenology at the three frontiers of the search for WIMP dark matter. For direct detection, unlike the Quark Portal case, dark matter particles do not directly couple to target nuclei at tree level. At one loop, the dark matter can couple to the photon through various electromagnetic moments, which generates the dominant interaction with the target nucleus. The latest LUX results from ref. [17] can constrain a large portion of parameter space for Dirac fermion or complex scalar dark matter. For indirect detection, dark matter particle annihilation can generate electrons or positrons with a harder spectrum than the Quark Portal case. Hence, the electron and positron flux measurement from AMS-02 in refs. [18, 19] becomes relevant for the Lepton Portal models. At colliders, the Quark Portal models have a larger signal production but also a larger QCD background. In the Lepton Portal models, the dark matter mediator particles can be pair produced via off-shell photons or $Z$ bosons. The corresponding collider signature is two same-flavor charged leptons plus missing transverse energy. Because both ATLAS and CMS collaborations at the LHC can make very good measurements of charged lepton momenta, the signature of dilepton plus missing transverse energy could serve as the discovery channel for dark matter particles. Therefore, we pay more attention to understanding and optimizing the key kinematic variables and work out the sensitivity at the $14 \mathrm{TeV}$ LHC.

Colliders can cover the light dark matter mass region beyond the direct and indirect detection sensitivity. This is simply due to different kinematics for different probes. For the three categories of dark matter particles: Majorana fermion, Dirac fermion and complex scalar, we have found that the $14 \mathrm{TeV}$ LHC has a much better reach than the direct detection experiments for the Majorana fermion and complex scalar cases. For the Majorana case, the dark matter scattering cross section is suppressed by the dark matter velocity and predicts a very small rate for direct detection experiments. For the complex scalar case, the dark matter fermion partner has a large production cross section at the LHC and a high discovery probability at the LHC.

Our paper is organized as follows. In section 2, we introduce the Lepton Portal class of simplified models. We determine the allowed parameter space for dark matter to be 
a thermal relic in section 3 . The direct detection will be covered in section 4 , where we perform loop-level calculations to determine the dark matter elastic scattering cross section. In section 5, we work out constraints on model parameter space from the AMS-02 positron and electron flux measurement. We then perform a collider study for the sensitivity at the $14 \mathrm{TeV}$ LHC with $100 \mathrm{fb}^{-1}$ and present summary plots in section 6 . We conclude in section 7 .

\section{A simplified dark matter model: the Lepton Portal}

In order for SM leptons to be a portal to the dark sector, there must be at least two particles, one fermion and one boson, in the dark sector. For simplicity, we assume that there is a $\mathcal{Z}_{2}$ symmetry under which the dark sector particles are odd which stabilizes dark matter. The lighter $\mathcal{Z}_{2}$ odd particle is the dark matter candidate. For the fermonic dark matter case, we will consider both Majorana and Dirac fermions because they have different annihilation and direct detection features. For the bosonic dark matter case, we only consider the complex scalar case, ignoring the real scalar case, which has suppressed direct detection rates [20]. In this paper, we only consider the right-handed leptons as the portal particles. The left-handed lepton case requires the dark matter partner to be a weak doublet for renormalizable couplings and hence more degrees of freedom. The extra mediator degree of freedom would be a singlet under the electromagnetic symmetry. The phenomenology associated with the charged component of the mediator is similar to that of a mediator coupling to right-handed leptons. There are additional potential collider signatures involving both mediators such as multi-lepton plus MET. In general, the weak doublet mediator models will be more constrained by collider searches than the models considered below.

For fermonic (Dirac or Majorana) dark matter, $\chi$, the partner is a scalar, $\phi$, with an electric charge +1 . The renormalizable operators for the dark matter coupling to the right-handed leptons are

$$
\mathcal{L}_{\text {fermion }} \supset \lambda_{i} \phi_{i} \bar{\chi}_{L} e_{R}^{i}+\text { h.c. },
$$

where $e^{i}=e, \mu, \tau$ are the charged leptons. The dark matter mass $m_{\chi}$ is smaller than its partner mass $m_{\phi}$ such that $\phi_{i}$ has a decay branching ratio $\operatorname{Br}\left(\phi^{i} \rightarrow \chi+\bar{e}^{i}\right)=100 \%$. For a complex scalar dark matter particle, $X$, the partner is a Dirac fermion, $\psi$, with electric charge -1 and the interactions

$$
\mathcal{L}_{\text {scalar }} \supset \lambda_{i} X \overline{\psi^{i}}{ }_{L} e_{R}^{i}+\text { h.c. }
$$

Again, we have $\operatorname{Br}\left(\psi^{i} \rightarrow X+e^{i}\right)=100 \%$.

To simplify our discussion, we define the Yukawa couplings in eqs. (2.1) and (2.2) to be in the charged-lepton mass eigenstates, so there are no new contributions to the flavor violating processes from the dark matter sector. This assumption can easily be arranged by implementing Minimal Flavor Violation (MFV) in the lepton sector [21]. Under the MFV assumption and to leading order in the lepton Yukawa matrices, the couplings 
could be made proportional to the charged lepton Yukawa matrix $Y_{\ell}$ or to combinations of the identity and $Y_{\ell}^{\dagger} Y_{\ell}$. Various model-dependent neutrino flavor structures could also be inserted, but they can be highly suppressed as in models of with Dirac neutrinos. Ultimately, MFV is sufficient in the lepton sector to fully eliminate contributions to lepton flavor violation. We defer a more complete study of various neutrino Yukawa insertion to future work.

In the following study, we will consider one flavor at one time. This assumption can easily be arranged for electron and tau coupling. For the muon case, it is trickier to arrange such a setup. In fact, the muon-like mediator would generally be degenerate with the electron mediator within MFV setups. Our results in that case give conservative, phenomenology-based sensitivity, while remaining agnostic to the flavor structure of corresponding full theories. Combinations of two or three flavors can be worked out based on the results for an individual flavor. For each flavor, we have only three parameters: the dark matter mass, its partner mass and the coupling strength. We will work out the standard dark matter phenomenology including thermal relic abundance, direct detection, indirect detection and collider searches, in sequence.

\section{Relic abundance}

Starting with the fermonic dark matter case, the main annihilation channel is $\bar{\chi} \chi \rightarrow \overline{e^{i}} e^{i}$ for Dirac fermion dark matter. The dominant contribution to the annihilation cross-section is

$$
\frac{1}{2}(\sigma v)_{\text {Dirac }}^{\chi \bar{\chi}}=\frac{1}{2}\left[\frac{\lambda^{4} m_{\chi}^{2}}{32 \pi\left(m_{\chi}^{2}+m_{\phi}^{2}\right)^{2}}+v^{2} \frac{\lambda^{4} m_{\chi}^{2}\left(-5 m_{\chi}^{4}-18 m_{\chi}^{2} m_{\phi}^{2}+11 m_{\phi}^{4}\right)}{768 \pi\left(m_{\chi}^{2}+m_{\phi}^{2}\right)^{4}}\right] \equiv s+p v^{2},
$$

where $v$ is the relative velocity of two dark matter particles and is typically $0.3 c$ at the freeze-out temperature and $10^{-3} c$ at present. We have neglected lepton masses and use $\lambda$ to represent $\lambda_{e}, \lambda_{\mu}, \lambda_{\tau}$ for different flavors. Throughout our calculation, we consider only coupling to one flavor at a time. The factor of $1 / 2$ in eq. (3.1) accounts for the fact that Dirac dark matter is composed of both a particle and an anti-particle. For Majorana fermion dark matter, the annihilation rate only contains a $p$-wave contribution at leading order in the limit of zero lepton masses

$$
(\sigma v)_{\text {Majorana }}^{\chi \chi}=v^{2} \frac{\lambda^{4} m_{\chi}^{2}\left(m_{\chi}^{4}+m_{\phi}^{4}\right)}{48 \pi\left(m_{\chi}^{2}+m_{\phi}^{2}\right)^{4}} \equiv p v^{2} .
$$

For complex scalar dark matter, the annihilation rate of $X X^{\dagger} \rightarrow \overline{e^{i}} e^{i}$ is also $p$-wave suppressed and given by

$$
\frac{1}{2}(\sigma v)_{\text {complex scalar }}^{X X^{\dagger}}=\frac{1}{2}\left[v^{2} \frac{\lambda^{4} m_{X}^{2}}{48 \pi\left(m_{X}^{2}+m_{\psi}^{2}\right)^{2}}\right] \equiv p v^{2} .
$$

Following the same relic abundance calculation in ref. [8], we show the parameter space for a relic abundant dark matter for Dirac fermion, Majorana fermion and complex scalar cases in figure 1 . We have neglected the co-annihilation effects when the mediator and 


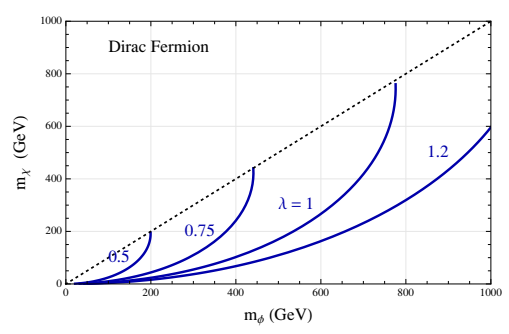

(a)

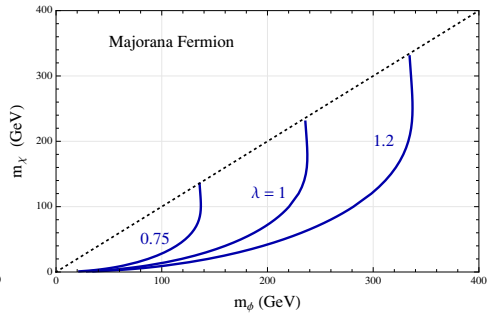

(b)

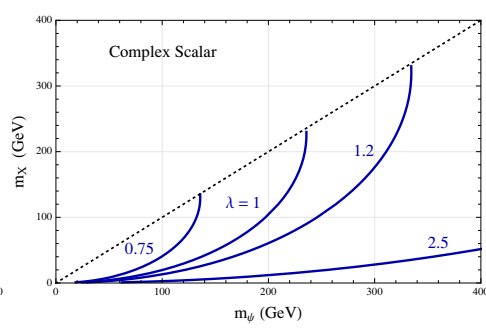

(c)

Figure 1. Parameter space of a thermal dark matter for (a) Dirac fermion, (b) Majorana fermion and (c) complex scalar.

dark matter masses are degenerate (see refs. [22, 23] for studies on the co-annihilation region in supersymmetry models). We expect that within the region where the mediator and DM are nearly degenerate, coannihilation effects will lead to a line in parameter space up to large dark matter masses where DM can be a thermal relic. While colliders have difficulty probing this region of parameter space (monojet plus MET searches will be the most sensitive ones), direct detection experiments should be sensitive. Unlike in the case of the quark portal, however, there is no resonant enhancement of the direct detection cross-section in the degenerate region. As one can see from figure 1, the Dirac fermion case has heavier allowed dark matter masses compared to the other two cases for a fixed value of $\lambda$.

\section{Dark matter direct detection}

Since the dark matter particle only interacts with leptons at tree-level, direct detection of dark matter in underground experiments requires either that dark matter scatter off electrons in the target at tree level [24] or off nucleons at one-loop level. Because of the electron wave-function suppression, the dominant contribution in Lepton Portal models still comes from one-loop process with a virtual photon coupling to nucleus. A representative Feynman diagram is shown in figure 2. There is also a similar diagram with a $Z$-boson exchange, but that process faces an additional $q^{4} / m_{Z}^{4}$ suppression and is negligible. Since DM is an SM gauge singlet within the lepton portal framework, no other tree-level interactions are possible.

Since dark matter couples to photons at one-loop level, we will first identify the relevant effective operators and then perform an explicit calculation to match the coefficients of the effective operators. To understand the physical meanings of those operators, we will also identify the dark matter electromagnetic moments for different operators in appendix A.

For the Dirac fermion case, there are two dimension-six operators ${ }^{1}$ generated at one

\footnotetext{
${ }^{1}$ Note that, in principle, there are chirally suppressed dimension-five magnetic dipole operators. Such operators are contained in the operator $\mathcal{O}_{1}^{\text {Dirac }}$ of eq. (4.1). Using the dark matter equation of motion, one can derive the coefficient of the nominally dimension-five magnetic-dipole operator, which is proportional to $m_{\chi} / m_{\phi}^{2}$.
} 


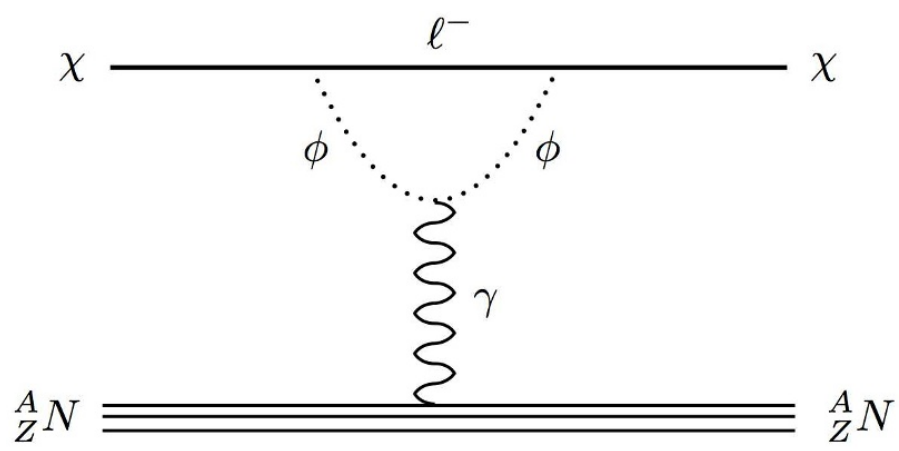

Figure 2. A representative Feynman diagram for dark matter scattering off nucleus via exchanging photon at loop level. Other diagrams can have the charged lepton connect to a photon.

loop by which dark matter intercts with photons. They are

$$
\mathcal{O}_{1}^{\text {Dirac }}=\left[\bar{\chi} \gamma^{\mu}\left(1-\gamma^{5}\right) \partial^{\nu} \chi+\text { h.c. }\right] F_{\mu \nu}, \quad \mathcal{O}_{2}^{\text {Dirac }}=\left[i \bar{\chi} \gamma^{\mu}\left(1-\gamma^{5}\right) \partial^{\nu} \chi+\text { h.c. }\right] F^{\alpha \beta} \epsilon_{\mu \nu \alpha \beta},
$$

which yield charge-charge interactions as the leading interactions between dark matter and nuclei [25]. These operators contain the charge radius, electromagnetic anapole, and magnetic dipole moments of the Dirac dark matter. For the Majorana fermion case, only one chiral structure of the bi-fermion part exists. It seems that one has two dimension-six operators at one-loop with the forms

$$
\mathcal{O}_{1}^{\text {Majorana }}=\left[-\bar{\chi} \gamma^{\mu} \gamma^{5} \partial^{\nu} \chi+\text { h.c. }\right] F_{\mu \nu}, \quad \mathcal{O}_{2}^{\text {Majorana }}=\left[i \bar{\chi} \gamma^{\mu} \partial^{\nu} \chi+\text { h.c. }\right] F^{\alpha \beta} \epsilon_{\mu \nu \alpha \beta} .
$$

However, one can use the Chisholm identity to prove that $\mathcal{O}_{2}^{\text {Majorana }}=-2 \mathcal{O}_{1}^{\text {Majorana }}$ (see appendix A for further details). ${ }^{2}$ Therefore, we only have a single dimension-six operator for the Majorana fermion case. This operator can be matched to the electromagnetic anapole moment of dark matter coupling to the current from the target in the non-relativistic limit (for general discussion about anapole dark matter see refs. [26, 27] and especially ref. [28] for clarifying a mistake in ref. [27].).

In the Lepton Portal model, the single-flavor contribution to the effective operator in the Lagrangian is calculated and is given by

$$
\mathcal{L} \supset c_{1} \mathcal{O}_{1}+c_{2} \mathcal{O}_{2}, \quad \text { with } \quad c_{1} \equiv \frac{-\lambda^{2} e}{64 \pi^{2} m_{\phi}^{2}}\left[\frac{1}{2}+\frac{2}{3} \ln \left(\frac{m_{e^{i}}^{2}}{m_{\phi}^{2}}\right)\right], \quad c_{2} \equiv \frac{-\lambda^{2} e}{64 \pi^{2} m_{\phi}^{2}} \frac{1}{4},
$$

for both Dirac and Majorana cases. ${ }^{3}$ For muon and tau cases, we use the masses for $m_{e^{i}}$. For the electron case, for which the lepton mass is below the exchange momentum of the scattering process, one should replace $m_{e^{i}}$ by the exchange momentum $|\vec{q}|$ with $\vec{q}^{2}=2 \mu_{\chi T}^{2} v^{2}(1-\cos \theta)=\mathcal{O}(10-100) \mathrm{MeV}$ depending on the dark matter mass. Here, $\mu_{\chi T}$ is the reduced mass of the dark matter-nucleus system; $\theta$ is the scattering angle in the center-of-mass frame; $v$ is the dark matter velocity in the lab frame.

\footnotetext{
${ }^{2}$ We thank Wai-Yee Keung for cross checking this point.

${ }^{3}$ We have checked our formulas against ref. [25] and agree with their calculation.
} 
For the Dirac dark matter case, neglecting the form factors on the dark matter side, we still have two different moments for photon coupling to the nucleus in the target. Since the charge and magnetic dipole moment parts have different form factors, we keep track of those parts in our calculation. For the spin-independent coupling to the charge of the nucleus, the differential scattering cross section in the recoil energy, $E_{R}=|\vec{q}|^{2} / 2 m_{T}$, at the leading order in $v^{2}$ is

$$
\frac{d \sigma_{T}^{E}}{d E_{R}}=\left[c_{1}^{2} e^{2} Z^{2} \frac{m_{T}}{2 \pi v^{2}}+c_{2}^{2} e^{2} Z^{2}\left(\frac{4 m_{\chi}^{2}}{\pi E_{R}}-\frac{2 m_{\chi}^{2} m_{T}}{\pi \mu_{\chi T}^{2} v^{2}}\right)\right] F_{E}^{2}\left(q^{2}\right),
$$

where $Z$ is the charge of the target nucleus and $F_{E}\left(q^{2}\right)$ is the electric form factor of the target nucleus. ${ }^{4}$ For the coupling to the nuclear magnetic dipole moment, we obtain a differential cross section

$$
\frac{d \sigma_{T}^{M}}{d E_{R}}=c_{2}^{2} e^{2} \frac{4}{\pi} \frac{m_{\chi}^{2}}{m_{T} v^{2}} \frac{m_{T}^{2} \lambda_{T}^{2}}{m_{N}^{2} \lambda_{N}^{2}} \frac{J_{T}+1}{3 J_{T}} F_{M}^{2}\left(q^{2}\right) .
$$

Here, $\lambda_{N}=e / 2 m_{N}$ is the nuclear magneton; $m_{N}$ is the nucleon mass; $\lambda_{T}$ is the target nucleus magnetic moment; $J_{T}$ is the spin of the target nucleus; $F_{M}\left(q^{2}\right)$ is the form factor of the nucleus magnetic dipole moment. For the Xenon element, the two most abundant and stable isotopes have $\lambda_{T} / \lambda_{N}\left({ }_{54}^{129} \mathrm{Xe}\right)=-0.778$ with $J_{T}\left({ }_{54}^{129} \mathrm{Xe}\right)=1 / 2$ and an abundance of $26.40 \%$ and $\lambda_{T} / \lambda_{N}\left({ }_{54}^{131} \mathrm{Xe}\right)=+0.692$ with $J_{T}\left({ }_{54}^{131} \mathrm{Xe}\right)=3 / 2$ and an abundance of $21.23 \%$ [29] (see ref. [30] for a collection of nuclear magnetic moments for more elements in direct detection experiments). Comparing eqs. (4.4), (4.5), one can see that the magnetic moment part is sub-leading compared to the charge part for a light dark matter because of the $m_{\chi} / m_{T}$ and $\left|c_{2} / c_{1}\right| \sim 1 / 40(1 / 20)$ suppression factors for muon(tau). Keeping the leading and first term in eq. (4.4), we have the same $v^{2}$ dependence as the spin-independent scattering. We obtain approximate results for dark matter-nucleus and dark matter-nucleon scattering cross sections as

$$
\sigma_{\chi T}=c_{1}^{2} e^{2} Z^{2} \frac{\mu_{\chi T}^{2}}{\pi}, \quad \sigma_{\chi N}=c_{1}^{2} e^{2} Z^{2} \frac{\mu_{\chi N}^{2}}{A^{2} \pi},
$$

where $\mu_{\chi N}$ is the reduced mass of the dark matter-nucleon system. Using the LUX result [17], we show the constrains on the model parameter space in figure 6 using $Z=54$ and $A=129$.

For the Majorana fermion case, the dimension-six operators in eq. (4.2) couple to the charge and the magnetic dipole moment of the nucleus. The differential cross section in $E_{R}$ is suppressed by an additional power of $v^{2}$ compared to the Dirac fermion case and leads a weak direct detection signals. The form for the charge part is

$$
\frac{d \sigma_{T}^{E}}{d E_{R}}=\left(c_{1}-2 c_{2}\right)^{2} e^{2} Z^{2} \frac{m_{T}}{4 \pi}\left(2-\frac{m_{T} E_{R}}{\mu_{\chi T}^{2} v^{2}}\right) F_{E}^{2}\left(q^{2}\right) .
$$

\footnotetext{
${ }^{4}$ Our result is different from ref. [25]. We don't have a term proportional $c_{1} c_{2}$ because the dark matter (a point-like particle) charge and magnetic-dipole moment parts should be summed together in the matrix element calculation and their $c_{1} c_{2}$ terms cancel each other.
} 
The dipole moment part has

$$
\frac{d \sigma_{T}^{M}}{d E_{R}}=\left(c_{1}-2 c_{2}\right)^{2} e^{2} \frac{1}{2 \pi} \frac{E_{R}}{v^{2}} \frac{m_{T}^{2} \lambda_{T}^{2}}{m_{N}^{2} \lambda_{N}^{2}} \frac{J_{T}+1}{3 J_{T}} F_{M}^{2}\left(q^{2}\right),
$$

which agrees with the results in ref. [28] and disagrees with ref. [27], which used the same form factors for charge and magnetic dipole interactions. For the typical direct detection experiments, one has the recoiled energy from a few $\mathrm{keV}$ to a hundred keV. Choosing a representative $E_{R}^{\text {ref }}=10 \mathrm{keV}$, we obtain the reference dark matter-nucleon scattering cross section

$$
\sigma_{\chi N}^{\mathrm{ref}}=\frac{\left(c_{1}-2 c_{2}\right)^{2} e^{2} Z^{2}}{2 \pi A^{2}} \frac{E_{R}^{\mathrm{ref}} m_{p}^{2}\left(m_{T}+m_{\chi}\right)^{2}}{m_{T}\left(m_{p}+m_{\chi}\right)^{2}} \approx 2 \times 10^{-49} \mathrm{~cm}^{2},
$$

for ${ }_{54}^{129} \mathrm{Xe}$ and the muon case with $m_{\chi}=50 \mathrm{GeV}, m_{\phi}=100 \mathrm{GeV}$ and $\lambda=1$. The current LUX results are not sensitive to this cross section. We therefore do not show the direct detection constraints on the Majorana fermion case in our plots.

For the complex scalar case, the dominant contribution can be related to the charge radius operator

$$
\mathcal{L} \supset C \partial^{\mu} X \partial^{\nu} X^{\dagger} F_{\mu \nu}
$$

with the matched coefficient as $C\left(m_{e^{i}}, m_{\psi}\right)$ and the formula

$$
C\left(m_{1}, m_{2}\right)=\frac{\lambda^{2} e}{16 \pi^{2}}\left[\frac{m_{1}^{4}-6 m_{1}^{2} m_{2}^{2}+m_{2}^{4}}{\left(m_{1}^{2}-m_{2}^{2}\right)^{3}}-\frac{4\left(m_{1}^{2}+m_{2}^{2}\right)\left(m_{1}^{4}-5 m_{1}^{2} m_{2}^{2}+m_{2}^{4}\right)}{3\left(m_{1}^{2}-m_{2}^{2}\right)^{4}} \ln \left(\frac{m_{1}}{m_{2}}\right)\right],
$$

where $C\left(m_{1}, m_{2}\right) \propto\left(m_{1}-m_{2}\right)$ in the limit of $m_{1}-m_{2} \ll 0$. In the limit of $m_{1} \ll m_{2}$, one has

$$
C\left(m_{1}, m_{2}\right)=-\frac{\lambda^{2} e}{16 \pi^{2} m_{2}^{2}}\left[1+\frac{2}{3} \ln \left(\frac{m_{1}^{2}}{m_{2}^{2}}\right)\right]
$$

The spin-independent dark matter-nucleus differential scattering cross section, at the leading order in $v^{2}$, is calculated to be

$$
\frac{d \sigma}{d E_{R}}=\frac{Z^{2} e^{2} C^{2}\left(m_{e^{i}}, m_{\psi}\right) m_{T}}{16 \pi v^{2}} F_{E}^{2}\left(q^{2}\right)
$$

which has the same $v^{2}$ dependence as the ordinary spin-independent scattering. We obtain the total scattering cross section and the averaged dark matter-nucleon cross sections

$$
\sigma_{X T}=\frac{Z^{2} e^{2} C^{2}\left(m_{e^{i}}, m_{\psi}\right) \mu_{X T}^{2}}{8 \pi}, \quad \sigma_{X N}=\frac{Z^{2} e^{2} C^{2}\left(m_{e^{i}}, m_{\psi}\right) \mu_{X N}^{2}}{A^{2} 8 \pi} .
$$

The constraints on the model parameter space from LUX [17] are shown in figure 7. 


\section{Dark matter indirect detection}

The indirect detection of dark matter tries to observe the excess of events in cosmic rays. If the dark matter annihilation cross section is not $p$-wave suppressed, this is the most efficient way to test the "WIMP miracle". For the three dark matter cases considered in this paper, we only have the Dirac fermion case with a large indirect detection signal. We therefore work out the relevant predictions in the Lepton Portal models for the Dirac fermion dark matter. We also note that we have not considered the case of a degenerate spectrum with co-annihilation. Future indirect detection results from CTA [31] could serve as the leading approach to uncover this region of parameter space as emphasized in ref. [32, 33].

The primary bounds on the Lepton Portal models from indirect detection come from measurements of the high-energy positron flux. Most astrophysical processes generate more electrons than positrons, while dark matter annihilations in the Lepton Portal model produce them in equal amounts, leading to a distinctive excess in the positron fraction, particularly at high energies for relatively heavy dark matter.

Several experiments have measured the positron fraction at high energies, but the cleanest measurement for the region of interest was performed by the AMS-02 experiment. They observed a rise in the positron fraction above $10 \mathrm{GeV}$ that cannot be conclusively explained by known astrophysical sources [18] (see ref. [34] for PAMELA results and ref. [35] for Fermi-LAT results). The leading candidate SM explanation for this excess at the time of this publication is the generation and acceleration of positrons in pulsars [36]. The possibility that this excess is due to annihilations or decays of dark matter particles remains allowed.

Portions of Lepton Portal parameter space are excluded by the AMS-02 data regardless of the origin of the positron fraction rise, simply by virtue of the fact that they produce a positron flux larger than observed. We determine the portion of parameter space excluded by the AMS-02 results in this section.

We begin by calculating the differential flux of positrons and electrons due to dark matter annihilation in the Lepton Portal model. For a given annihilation cross-section, the flux is given by [37]

$$
\Phi_{e^{ \pm}}(E)=B \frac{v_{e}}{4 \pi b(E)} \frac{1}{2}\left(\frac{\rho_{\odot}}{M_{\mathrm{DM}}}\right)^{2} \int_{E}^{M_{\mathrm{DM}}} d E^{\prime} f_{\mathrm{inj}, e^{ \pm}}\left(E^{\prime}\right) I\left[\lambda_{D}\left(E, E^{\prime}\right)\right] .
$$

The particle physics inputs to this calculation are encoded entirely in the dark matter mass, $M_{\mathrm{DM}}$, the injection spectrum, $f_{\text {inj }}$, and the electron/positron velocity, $v_{e} \approx c$. The dark matter injection spectrum is given by

$$
f_{\mathrm{inj}, e^{ \pm}}(E)=\sum_{k}\langle\sigma v\rangle_{k} \frac{d N_{e^{ \pm}}^{k}}{d E},
$$

where the sum is over processes with an electron/positron in the final state, $\langle\sigma v\rangle_{k}$ is the thermally averaged cross-section for annihilation via process $k$, and $d N_{e^{ \pm}}^{k} / d E$ is the expected number of electrons/positrons with energy between $E$ and $E+d E$ produced by 
the annihilation. For the case $\mathrm{DM}+\mathrm{DM} \rightarrow e^{+} e^{-}$, using the fact that the annihilations occur between non-relativistic DM particles, we find

$$
\frac{d N_{e^{+}}^{k}}{d E}=\frac{d N_{e^{-}}^{k}}{d E}=\delta\left(E-M_{\mathrm{DM}}\right)
$$

The muon and tau cases have been studied in ref. [38]. For the muon case,

$$
\frac{d N_{e^{+}}^{k}}{d E}=\frac{d N_{e^{-}}^{k}}{d E}=\frac{1}{3 M_{\mathrm{DM}}}\left(5-9 x^{2}+4 x^{3}\right) \times \theta\left(M_{\mathrm{DM}}-E\right),
$$

where $x=E / M_{\mathrm{DM}}$ and $\theta$ is the Heaviside theta function. For the tau case, the spectrum is generated using Pythia [39] and is fitted by [38]

$$
\begin{array}{r}
\frac{d N_{e^{+}}^{k}}{d E}=\frac{d N_{e^{-}}^{k}}{d E}=\frac{1}{M_{\mathrm{DM}}}\left(e^{-97.716 x^{5}+223.389 x^{4}-193.748 x^{3}+82.595 x^{2}-22.942 x+2.783}\right. \\
\left.+e^{-15.575 x^{3}+15.79 x^{2}-18.083 x+0.951}\right) \theta\left(M_{\mathrm{DM}}-E\right) .
\end{array}
$$

The remaining factors in eq. (5.1) are purely astrophysical. $B$, taken to be 1 , is a boost factor that accounts for possible local clumping of dark matter. The energy loss coefficient $b(E)=E^{2} /\left(\mathrm{GeV} \cdot \tau_{E}\right)$ with $\tau_{E}=10^{16} \mathrm{~s}$, is defined by the diffusion equation

$$
\frac{\partial f}{\partial t}-K(E) \nabla^{2} f-\frac{\partial}{\partial E}[b(E) f]=Q,
$$

where $K(E)$ is the diffusion coefficient and $Q$ is the annihilation injection term. $\rho_{\odot}$ is the local dark matter density. $I$ is the "halo function", depending on the diffusion length $\lambda_{D}$. All of these quantities are described and fit to functions in [37, 40] for a variety of assumptions ranging from conservative to optimistic. In this study, we use the flux as determined by the min, med, and max set of assumptions from [40] to represent minimal, medium, and maximal fluxes attainable by varying the astrophysical assumptions.

We now determine the excluded regions of Lepton Portal parameter space assuming that the observed flux is enitrely due to SM processes. To be conservative regarding astrophysical positron sources, we determine that a model is excluded if it predicts a total positron flux more than $2 \sigma$ in excess of that measured by AMS-02 in any energy bin (see refs. [41, 42] for model-independent constraints). The total number of positrons predicted by AMS-02 is given by the product of the fraction spectrum [18] and the $e^{-}+e^{+}$ spectrum [19]

$$
\left.\frac{d \Phi_{e^{+}}}{d E}\right|_{\mathrm{AMS}}=\left.f_{e^{+}, \mathrm{AMS}}(E) \frac{d \Phi_{e^{-+} e^{+}}}{d E}\right|_{\mathrm{AMS}}
$$

Majorana fermion and complex scalar dark matter cases have a velocity-suppressed annihilation cross-section, ensuring that the indirect detection signal is too small to be observed. For Dirac fermion dark matter, non-zero $s$-wave annihilation leads to constraints from AMS-02. The formula for the annihilation cross-section in the non-relativistic limit is given by eq. (3.1) for a Dirac Fermion by neglecting the $p$-wave parts. As a benchmark, we also take the coupling $\lambda=1$. The resulting constraints under the conservative set of assumptions are shown in figure 3 . One can see that our conservative constraints require 


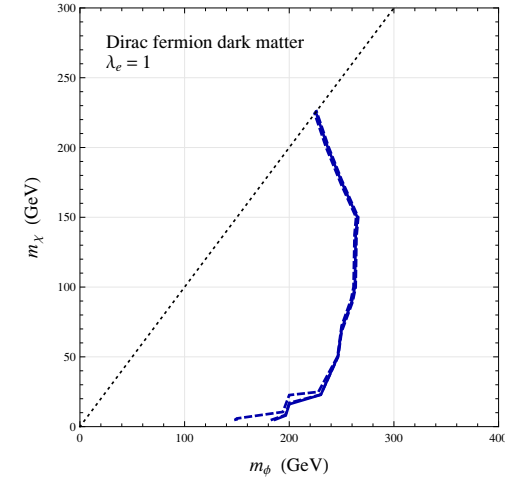

(a)

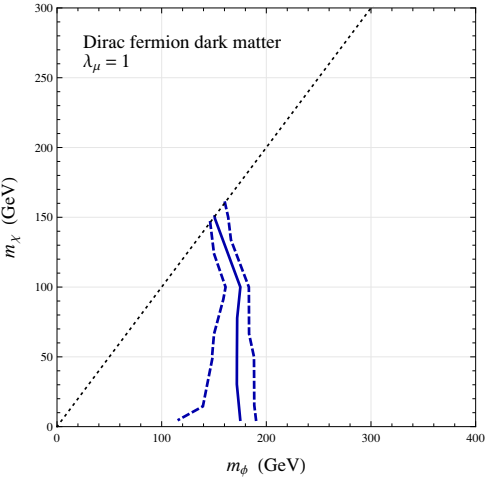

(b)

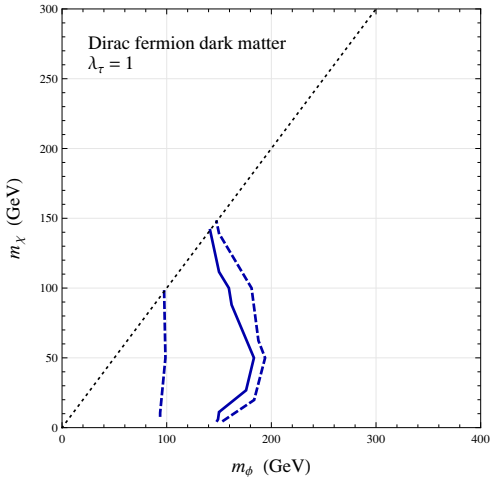

(c)

Figure 3. Indirect detection constraints on the Lepton Portal model for the Dirac fermion dark matter with coupling to (a) electrons; (b) muons; (c) taus. The left and right outer dashed lines represent the "minimal" and "maximal" astrophysical assumptions, respectively. The middle and solid line represents the "medium" astrophysical assumption.
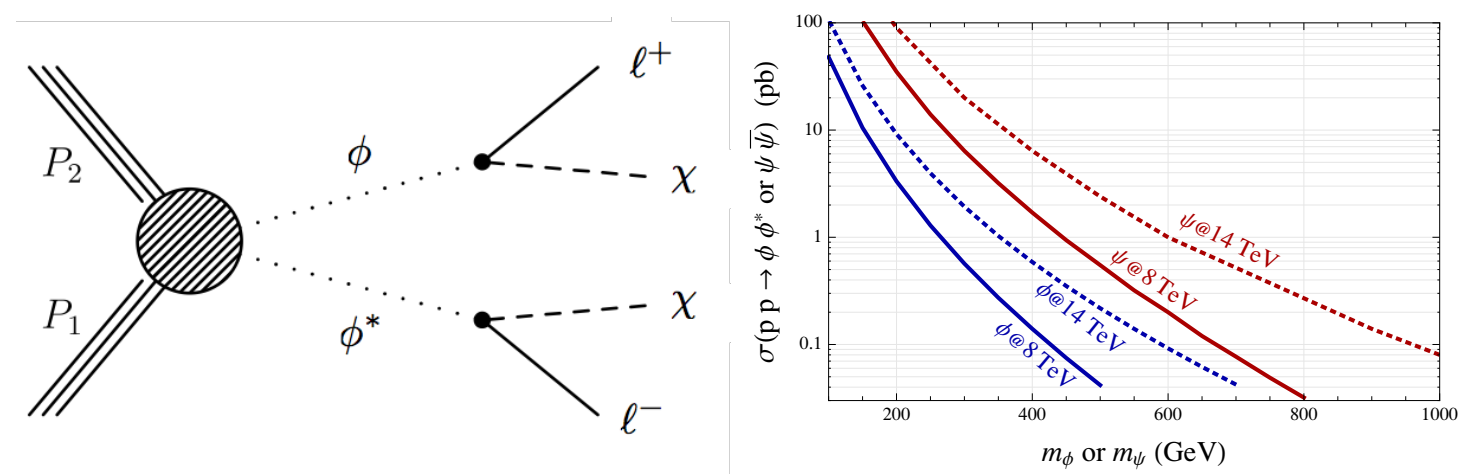

Figure 4. Left panel: Feynman diagram for the complex scalar mediator production and decay in the fermion dark matter models. Right panel: the production cross sections for the complex scalar and vector-like fermion mediators at the LHC.

the mediator masses to be above $100-300 \mathrm{GeV}$ for different flavor and propagation model assumptions. For the electron coupling case, the limits for the three different propagation models are similar to each other. This is because the electron/positron propagation difference decreases at an energy close to the dark matter mass and the constraints from AMS-02 mainly come from high energy bins.

\section{Collider constraints and searches}

At hadron colliders, the signature of Lepton Portal models comes from pair productions of the mediator via the Drell-Yan process. The produced mediator particles then decay into the dark matter particles plus leptons. The signature at hadron colliders is thus same-flavor, opposite-sign dilepton plus missing transverse energy, which is also the standard signature for searching for sleptons in the MSSM at colliders. We show the production and decay processes in the left panel of figure 4 for a complex scalar mediator. In the right-panel of 
figure 4 , we show the production cross sections of mediators, $\phi$ and $\psi$, for different masses at the LHC with both $8 \mathrm{TeV}$ and $14 \mathrm{TeV}$ center of mass energy. The $\phi+\phi^{*}$ production cross section is the same as a single-flavor right-handed slepton in MSSM [43, 44]. In the complex scalar dark matter case, the fermion mediator can be thought as a vectorlike fermion with the same electroweak quantum number as the right-handed electron. Its production cross sections are much larger than the scalar mediator one with the same mass. We will show later that the discovery sensitivity for this case is much better than the scalar mediator case.

Both ATLAS and CMS colaborations have searches for new physics in the $\ell^{+} \ell^{-}+$MET channel. The latest results from ATLAS with $20.3 \mathrm{fb}^{-1}$ at $8 \mathrm{TeV}$ have constrained the selectron and smuon masses to be above around $240 \mathrm{GeV}$ [45] for a light neutralino mass by summing the signal events from both selectron and smuon. For the Lepton Portal model with coupling only to a single flavor lepton, the signal production cross section is reduced by a factor of two. As a result, the constraint on the mediator mass is weaker and is around $170 \mathrm{GeV}$. A similar result has been obtained by the CMS collaboration [46], although different kinematic variables were used. The CMS collaboration has used $M_{\mathrm{CT} \perp}$ [47], which is related to the contransverse mass $M_{\mathrm{CT}}$ [48] (see also ref. [49] for the super-razor variable). On the other hand, the ATLAS collaboration has used the $M_{T 2}$ [50-53] variable to reduce the SM backgrounds (see also refs. [54-57] for recent applications on searching for stops). In our analysis, we concentrate on following the analysis of the ATLAS collaboration and use the $M_{T 2}$ variable to explore the discovery and exclusion sensitivities at both $8 \mathrm{TeV}$ and $14 \mathrm{TeV}$ LHC.

Other than the basic cuts on selecting the objects, the ATLAS searches have required two leptons with opposite signs and either the same or different flavors. They also veto events with a jet above $20 \mathrm{GeV}$, events with $\left|m_{\ell \ell}-m_{Z}\right|<10 \mathrm{GeV}$ and events with $M_{T 2}<$ 90(110) GeV. After those cuts, the main backgrounds are from diboson productions. The dilepton $M_{T 2}$ variable will be the most sensitive one for searching for higher mediator masses at the $14 \mathrm{TeV}$. It is defined as

$$
M_{T 2}=\min \left\{\bigcup_{\vec{p}_{1}^{T}+\vec{p}_{2}^{T}=\vec{E}_{T}^{\text {miss }}} \max \left[M_{T}\left(\vec{p}_{\ell_{1}}, \vec{p}_{1}^{T}\right), M_{T}\left(\vec{p}_{\ell_{2}}, \vec{p}_{2}^{T}\right)\right]\right\},
$$

with the transverse mass in terms of the lepton momentum $\vec{p}_{\ell_{i}}$ and the guessed missing particle (massless) transverse momentum $\vec{p}_{i}^{T}$. As we know from the discovery of the $W$ gauge boson, the transverse mass of the electron and neutrino is bounded from above by the $W$ gauge boson mass [58-61]. Imposing a cut on $M_{T 2}$ to be above the $W$ gauge boson mass can therefore dramatically reduce the dominant diboson backgrounds. The tail of the dilepton $M_{T 2}$ becomes the leading background, especially for a heavy mediator mass, as can be seen in figure 5. To estimate the current bounds on this model, we calculate LO cross-sections for the full process using MadGraph [62] using a model constructed by FeynRules [63]. The events are showered and hadronized using Pythia [39], then the hadrons are clustered into jets using PGS [64]. 

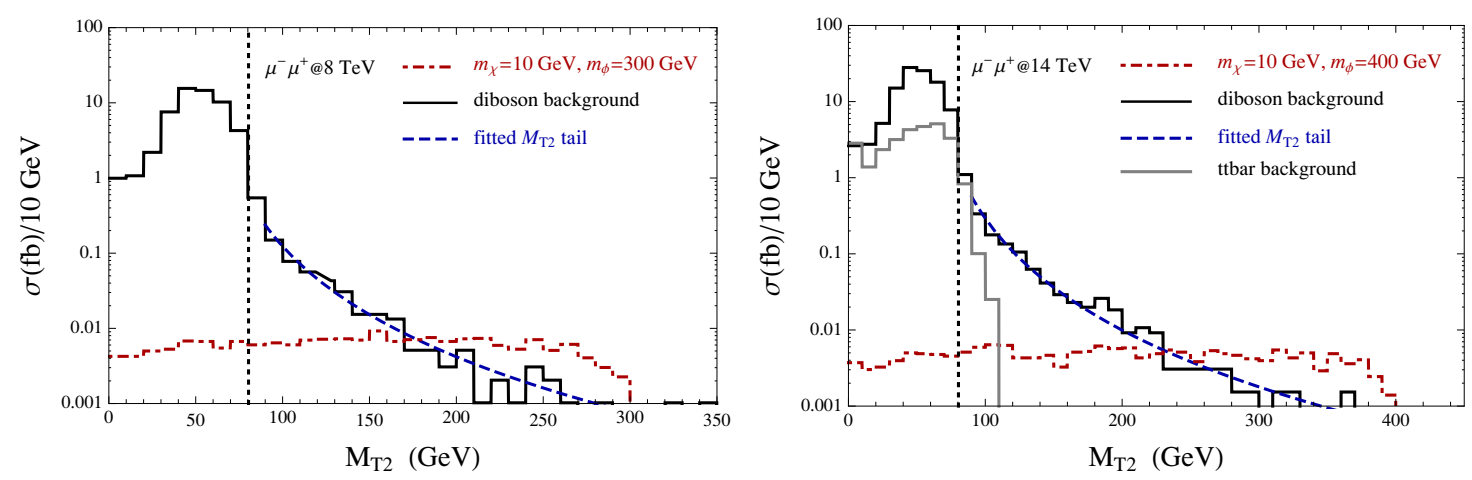

Figure 5. Left panel: the dilepton $M_{T 2}$ distributions for the diboson background and the signal events. The missing particle mass is assumed to be zero. The blue and dashed line is from the fitted function in eq. (6.2) with $\eta=2.0$. The vertical and dotted line indicates the reference $W$ gauge boson mass. Right panel: the same as the left one but for the $14 \mathrm{TeV}$ LHC together with the $t \bar{t}$ background. The same value $\eta=2.0$ is used for the fit function of eq. (6.2).

Motivated by the method of measuring the $W$ gauge boson width using the transverse tail distribution [61,65], we suspect that the tail of $M_{T 2}$ should be generated from off-shell $W$ gauge bosons and could follow the general Breit-Wigner distribution. We introduce the following parametrical function to fit the tail distribution

$$
F\left(M_{T 2}\right)=\frac{N_{0}}{\left[\eta M_{T 2}^{2}-M_{W}^{2}\right]^{2}+\eta^{2} M_{T 2}^{4} \Gamma_{W}^{2} / M_{W}^{2}} .
$$

Here, $N_{0}$ is the overall normalization and $\eta>1$ is suggested by the fact that the invariant mass of the $W$ gauge boson propagator is above the corresponding transverse mass. In figure 7, one can see that this Breit-Wigner distribution fits the tail pretty well. With a better understanding of the main background, the discovery reach of Lepton Portal dark matter can be extended.

We simulate the signal and background events at the $14 \mathrm{TeV}$ LHC and work out the $90 \%$ CL exclusion region on the model parameter space for $100 \mathrm{fb}^{-1}$ luminosity in the left panel of figure 6 and figure 7 for fixed $\lambda=1$. Specifically, for a given mass point, we have calculated the sensitivities for three different cuts: $M_{T 2} \geq 100,200,300 \mathrm{GeV}$ and chosen the most sensitive one as the potential reach. We also translate the LHC reach into the potential constraints on the dark matter-nucleon scattering cross section in the right panel of figure 6 and figure 7. For both Dirac fermion and complex scalar dark matter cases, the LHC searches have a better sensitivity for a light dark matter with a mass below $10 \mathrm{GeV}$. For the complex scalar dark matter case, the LHC has a better reach than direct detection experiments with dark matter masses up to around $500 \mathrm{GeV}$. This is due to the large production cross sections of vector-like fermion mediators at the LHC. The LHC reaches for the electron and muon cases are not different significantly from each other. The electron case has a larger acceptance and hence a better limit.

The collider constraints for the Majorana fermion dark matter case are identical to the Dirac fermion case, since the mediator production cross section determines the sen- 

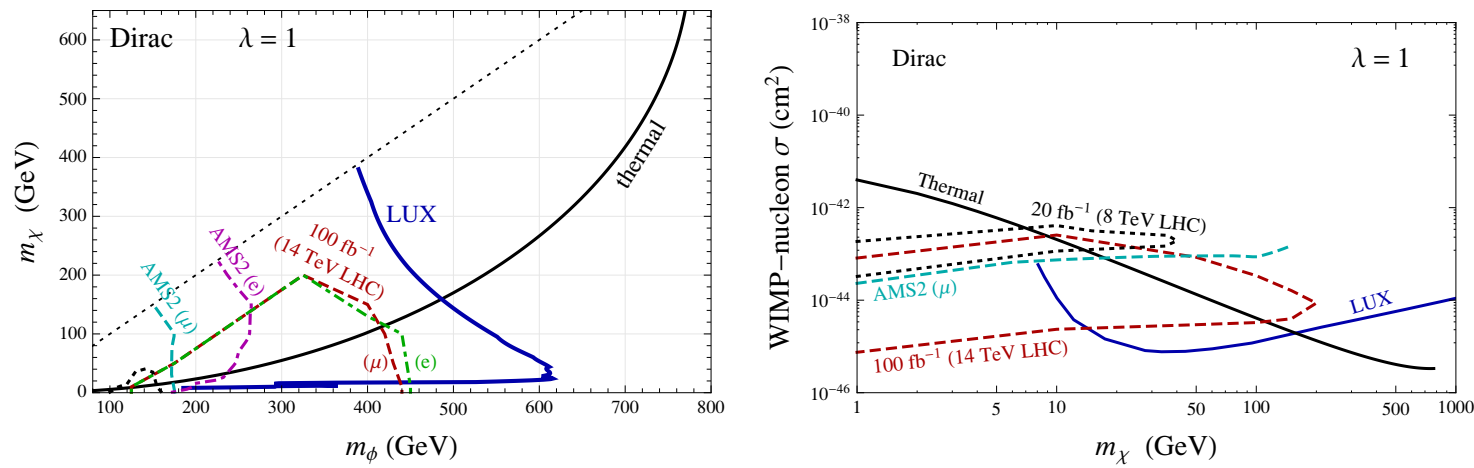

Figure 6. Left panel: the constraints on the dark matter and its mediator masses for the Dirac fermion case. The dotted and black line is the current constraint on the muon case from the $8 \mathrm{TeV}$ LHC with $20 \mathrm{fb}^{-1}$ [45]. Right panel: the dark matter-nucleon scattering cross section as a function of dark matter mass from different searches.
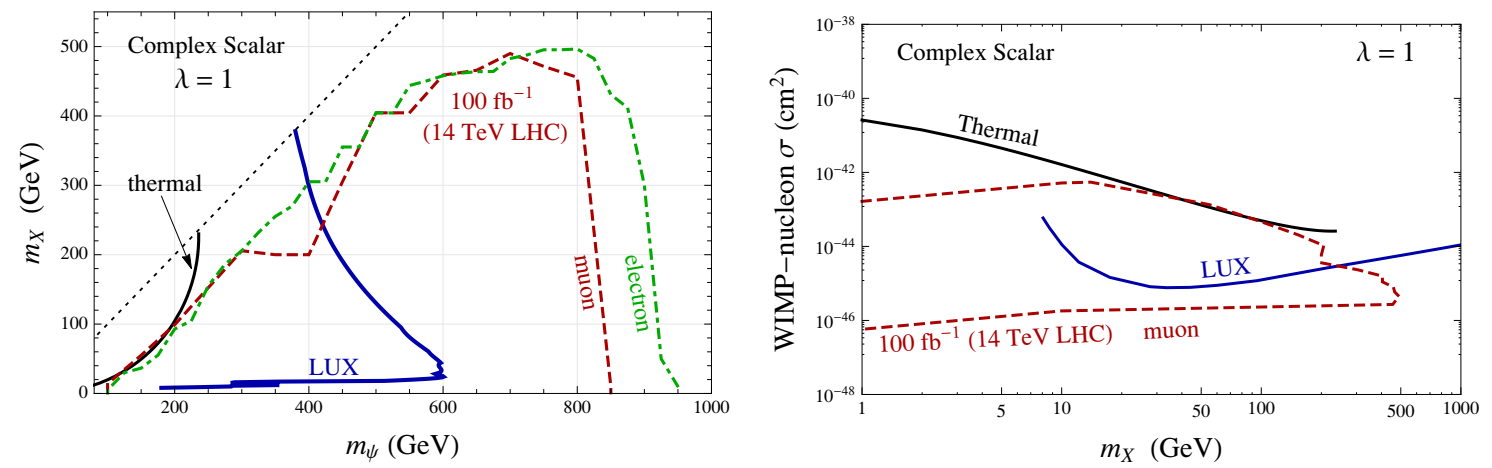

Figure 7. The same as figure 6 but for the complex scalar case. Because of the $p$-wave suppression of the dark matter annihilation cross section, the indirect detection constraints become very weak and are not shown here.

sitivity. As discussed in eq. (4.9), the direct detection cross section is very small for the Majorana fermion case. The indirect detection is $p$-wave suppressed or suppressed by $\mathcal{O}\left(v^{2} / c^{2} \approx 10^{-6}\right)$. The collider search is the most relevant one and can probe a large region of unexplored parameter space.

\section{Discussion and conclusions}

We want to first emphasize the importance of colliders for discovering or excluding the Lepton Portal dark matter. The signature with the same-flavor and opposite-sign dilepton plus missing energy is a pretty clean one. The $M_{T 2}$ cut can be imposed to make almost background free. As a result, the discovery reach is purely determined by the signal production cross section times the acceptance. For a large mass splitting between dark matter and its partner, the signal acceptance is large, so the discovery reach is limited by the signal cross section. From figure 4, one can see a large increase of the mediator production 
cross sections from $8 \mathrm{TeV}$ to $14 \mathrm{TeV}$ and a discovery of dark matter signals at the LHC may happen in the near future.

In our analysis, we have considered both the electron and muon cases and neglected the tau lepton case. We anticipate a slightly weaker limit from the LHC because of the tau-tagging and mis-tagging efficiencies. Another parameter region that we have ignored is the co-annihilation region. The collider searches become less sensitive because the leptons from the mediator decays are either too soft to pass the basic cuts or generate insufficient $M_{T 2}$ and would be buried in the SM backgrounds. In the extremely degenerate region, one could include an additional jet, photon, $W$ and $Z$ gauge bosons from initial state radiation to gain sensitivity.

In summary, we have studied Lepton Portal dark matter for three cases: Majorana fermion, Dirac fermion and complex scalar dark matter. For direct detection, the majorana fermion case has a very small predicted event rate because of the leading operator of the dark matter coupling to photon has an additional velocity suppression. On the other hand, the direct detection signals for the Dirac fermion and complex scalar cases are not suppressed. In terms of indirect detection, since only the Dirac fermion case has non-zero $s$-wave annihilation, AMS-02 has the best coverage for its model parameter space. At colliders, the LHC has better reaches for the light dark matter mass region than the direct detection experiments. For the complex scalar case, the $14 \mathrm{TeV}$ LHC with $100 \mathrm{fb}^{-1}$ can cover mediator masses up to $800 \mathrm{GeV}$ and provides a constraint on spin-independent dark matter-nucleon scattering cross section as low as $2 \times 10^{-46} \mathrm{~cm}^{2}$ for dark matter masses up to $500 \mathrm{GeV}$ and a unit coupling.

\section{Acknowledgments}

We thank Matthew Buckley, Zackaria Chacko, Spencer Chang and especially Wai-Yee Keung for useful discussions and comments. J. Berger would like to thank the Aspen Center for Theoretical Physics for their hospitality during the early stages of this work. Y. Bai is supported by the U. S. Department of Energy under the contract DE-FG-0295ER40896. SLAC is operated by Stanford University for the US Department of Energy under contract DE-AC02-76SF00515.

\section{A Non-relativistic correspondence of photon couplings}

At $v=0$, it is well known that there are only four ways in which a particle with spin $\vec{S}$ can couple to the electromagnetic field: charge operator $\Phi$, electric dipole moment $e \vec{S} \cdot \vec{E}$, magnetic dipole moment $e \vec{S} \cdot \vec{B}$, and anapole moment $e \vec{S} \cdot(\nabla \times \vec{B}) .{ }^{5}$ The properties of these operators under $C, P$ and $T$ are shown for future reference in table 1.

In the non-relativistic limit, any operator coupling $\bar{\chi}, \chi$, and $A^{\mu}$ should reduce to one or more of the above forms, up to corrections of $\mathcal{O}\left(\left[\nabla^{2}\right]^{i}\right)$ (radius corrections) and

\footnotetext{
${ }^{5}$ In principle, there could also be magnetic monopoles, in concert with "electric" anapoles. Magnetic monopoles violate $P$ and $T$. Electric anapoles violate $C$ and $T$. Without magnetic monopoles, there is no operator that violates $C$ and $T$.
} 


\begin{tabular}{|c|ccc|}
\hline Operator & $C$ & $P$ & $T$ \\
\hline$e \Phi$ & + & + & + \\
$e \vec{S} \cdot \vec{E}$ & + & - & - \\
$e \vec{S} \cdot \vec{B}$ & + & + & + \\
$e \vec{S} \cdot(\nabla \times \vec{B})$ & - & - & + \\
\hline
\end{tabular}

Table 1. $C, P, T$ properties of the non-relativistic couplings to photons.

$\mathcal{O}\left([\vec{v}]^{i}\right)$ which are fixed by Lorentz invariance. Based on the $C, P$ and $T$ properties of a given operator, one can determine which operator contributes. For any non-renormalizable operator, there cannot be a direct correspondence to $\Phi$, since gauge invariance demands dependence on $\vec{E}$ and $\vec{B}$ only. There may, however, still be charge radius terms from $\nabla \cdot \vec{E}=\nabla^{2} \Phi$.

Coming back to the operators $\mathcal{O}_{1}$ and $\mathcal{O}_{2}$ from eq. (4.1), we further break these operators up to highlight their contributions from operators with different $C, P$ and $T$ properties. We define

$$
\begin{aligned}
& e \mathcal{O}_{1}^{V}=e\left(\bar{\chi} \gamma^{\mu} \partial^{\nu} \chi+\text { h.c. }\right) F_{\mu \nu} \\
& e \mathcal{O}_{1}^{A}=-e\left(\bar{\chi} \gamma^{\mu} \gamma^{5} \partial^{\nu} \chi+\text { h.c. }\right) F_{\mu \nu} \\
& e \mathcal{O}_{2}^{V}=e \epsilon_{\mu \nu \alpha \beta}\left(i \bar{\chi} \gamma^{\mu} \partial^{\nu} \chi+\text { h.c. }\right) F^{\alpha \beta} \\
& e \mathcal{O}_{2}^{A}=-e \epsilon_{\mu \nu \alpha \beta}\left(i \bar{\chi} \gamma^{\mu} \gamma^{5} \partial^{\nu} \chi+\text { h.c. }\right) F^{\alpha \beta} .
\end{aligned}
$$

Then operators $\mathcal{O}_{1}^{V}$ and $\mathcal{O}_{2}^{A}$ have the same $C, P$ and $T$ properties of a charge or a magnetic dipole operator, while $\mathcal{O}_{1}^{A}$ and $\mathcal{O}_{2}^{V}$ have the properties of an anapole. $\mathcal{O}_{1}^{V}$ can easily be rewritten using integration by parts as $\bar{\chi} \gamma^{\mu} \chi \partial_{\nu} F^{\mu \nu}=J_{\chi}^{\mu} \partial_{\nu} F^{\mu \nu}$, making the correspondence to the charge radius operator evident. $\mathcal{O}_{2}^{A}$, on the other hand, contains the axial current which is proportional to spin in the non-relativistic limit; it maps onto a magnetic dipole operator. To order $v^{0}$, we then have

$$
\begin{aligned}
& e \mathcal{O}_{1}^{V} \approx e \xi^{\dagger} \xi \nabla \cdot \vec{E} \\
& e \mathcal{O}_{1}^{A} \approx-e \xi^{\dagger} \vec{S} \xi \cdot(\nabla \times \vec{B}), \\
& e \mathcal{O}_{2}^{V} \approx 2 e \xi^{\dagger} \vec{S} \xi \cdot(\nabla \times \vec{B}), \\
& e \mathcal{O}_{2}^{A} \approx-4 m e \xi^{\dagger} \vec{S} \xi \cdot \vec{B},
\end{aligned}
$$

where $\xi$ is the fermion wave-function, such that $e \bar{\xi} \xi$ is the charge density and $e \xi^{\dagger} \vec{S} \xi$ is the magnetization.

It may be further worth noting that these operators are not all independent. We have the following exact identities:

$$
2 \mathcal{O}_{1}^{A}+\mathcal{O}_{2}^{V}=0, \quad 2 \mathcal{O}_{1}^{V}+\mathcal{O}_{2}^{A}=2 m \mathcal{O}_{\text {dipole }}
$$

where $\mathcal{O}_{\text {dipole }}=\bar{\chi} \sigma^{\mu \nu} \chi F_{\mu \nu}$ is the dimension-five anomalous magnetic moment operator. These are obtained using gamma matrix identities and the Dirac equation. 


\section{B Lepton $g-2$}

In this appendix, we consider additional contributions to the lepton anomalous magnetic moments, which could also set constraints on our model parameters. Among the different flavors, the one that provides the most stringent constraint is the $a_{\mu}=(g-2)_{\mu} / 2$. On the other hand, there is a disagreement of more than $3 \sigma$ between the theoretical prediction and the experimental measurement on this quantity. The latest analysis of the hadronic contributions gives an SM prediction of [66]

$$
a_{\mu}^{\mathrm{SM}}=(11659182.8 \pm 4.9) \times 10^{-10},
$$

while the experimental measured value is higher and is $[67,68]$

$$
a_{\mu}^{\mathrm{EXP}}=(11659208.9 \pm 6.3) \times 10^{-10} .
$$

The difference is

$$
a_{\mu}^{\mathrm{EXP}}-a_{\mu}^{\mathrm{SM}}=(26.1 \pm 8.0) \times 10^{-10},
$$

which corresponds to a $3.3 \sigma$ discrepancy (see ref. [69] for a recent review and lattice QCD calculations for the SM prediction).

The lepton-portal dark matter could explain such a discrepancy. We check both parameter space that can fit the data and are allowed by the $a_{\mu}$ data. For Majorana (also for Dirac) fermion dark matter, the calculation has been done in the MSSM. The loop diagram from the dark matter and its partner has a negative contribution to $a_{\mu}$ as [70,71]

$$
\delta a_{\mu}^{(\chi, \phi)}=-\frac{\lambda^{2} m_{\mu}^{2}}{16 \pi^{2} m_{\phi}^{2}}\left[\frac{1-6 x+3 x^{2}+2 x^{3}-6 x^{2} \ln x}{6(1-x)^{4}}\right],
$$

with $x \equiv m_{\chi}^{2} / m_{\phi}^{2}$. In the region with degenerate masses, $x=1$, the part in the brackets becomes $1 / 12$. We show a few contours in the $m_{\chi}-m_{\phi}$ plane in the left panel of figure 8 for the fixed Yukawa coupling $\lambda=1$. Although the fermionic dark matter case cannot explain the $(g-2)_{\mu}$ anomaly, the dark matter contribution does not dramatically increase the discrepancy for a modest $\lambda$.

For the complex scalar dark matter case, the loop diagram from dark matter and its partner gives a positive contribution to $a_{\mu}$, which is given by

$$
\delta a_{\mu}^{(X, \psi)}=\frac{\lambda^{2} m_{\mu}^{2}}{16 \pi^{2} m_{X}^{2}}\left[\frac{2+3 x-6 x^{2}+x^{3}+6 x \ln x}{6(1-x)^{4}}\right],
$$

with $x \equiv m_{X}^{2} / m_{\psi}^{2}$. In the limit of $x=1$, the value in the bracket becomes $1 / 12$. With a large value of the coupling $\lambda=2.5$ and a light dark matter partner mass around $150 \mathrm{GeV}$, we show that the $(g-2)_{\mu}$ anomaly can be explained by the dark matter contribution in the right panel of figure 8. However, the LUX results have significantly constrained this $(g-2)_{\mu}$-favored region. 

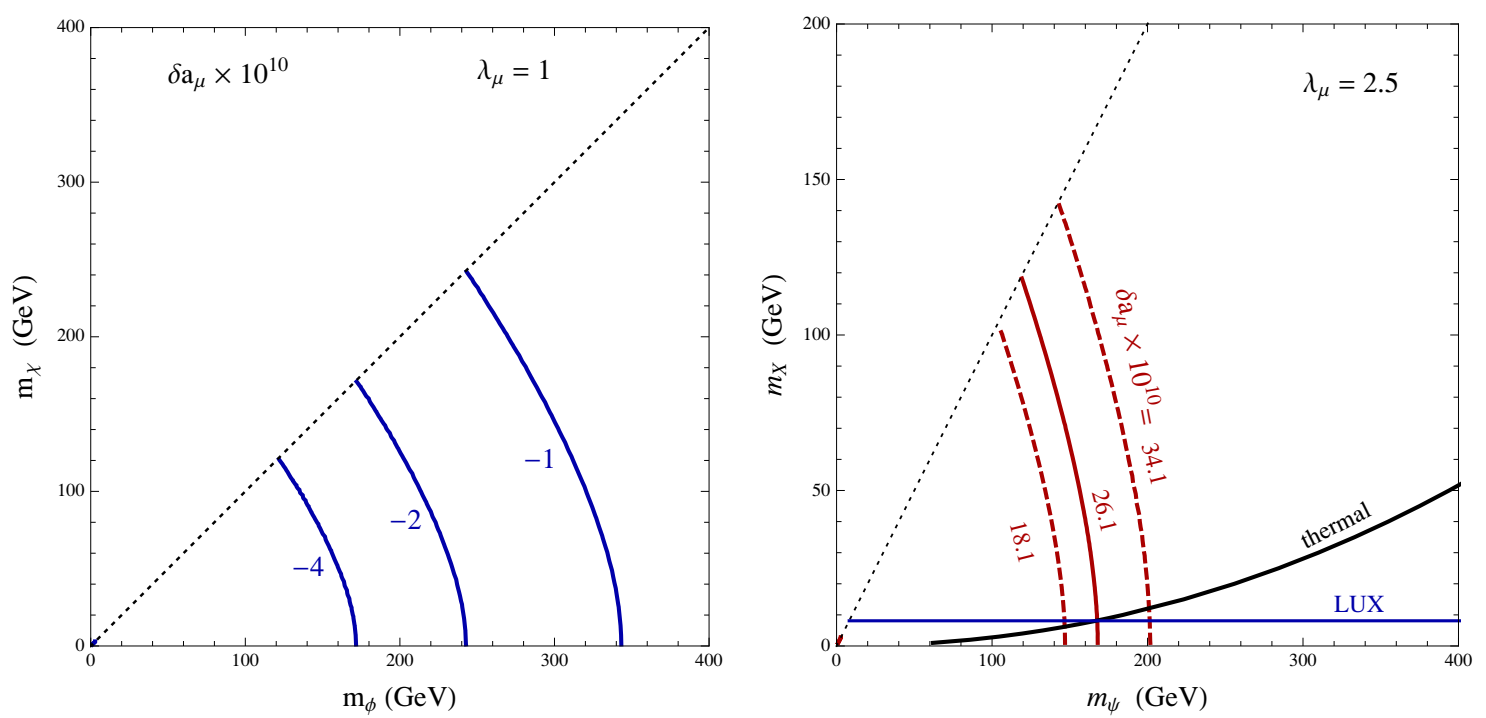

Figure 8. Left panel: the contours of dark matter contributions to $\delta a_{\mu}$ in the fermionic dark matter case. Right panel: for the complex scalar dark matter model, the solid line indicates parameter space giving a contribution to $(g-2)_{\mu}$ equal to the central value of the discrepancy. The two dashed lines are the one sigma boundaries from eq. (B.3). The region above the blue line is excluded by the direct detection results from LUX.

Open Access. This article is distributed under the terms of the Creative Commons Attribution License (CC-BY 4.0), which permits any use, distribution and reproduction in any medium, provided the original author(s) and source are credited.

\section{References}

[1] H.E. Haber and G.L. Kane, The Search for Supersymmetry: Probing Physics Beyond the Standard Model, Phys. Rept. 117 (1985) 75 [INSPIRE].

[2] G. Jungman, M. Kamionkowski and K. Griest, Supersymmetric dark matter, Phys. Rept. 267 (1996) 195 [hep-ph/9506380] [INSPIRE].

[3] M.W. Cahill-Rowley, J.L. Hewett, A. Ismail and T.G. Rizzo, More energy, more searches, but the phenomenological MSSM lives on, Phys. Rev. D 88 (2013) 035002 [arXiv: 1211.1981] [INSPIRE].

[4] M. Cahill-Rowley et al., Complementarity and Searches for Dark Matter in the pMSSM, arXiv:1305.6921 [INSPIRE].

[5] E.W. Kolb and M.S. Turner, The Early Universe, Front. Phys. 69 (1990) 1.

[6] S. Chang, R. Edezhath, J. Hutchinson and M. Luty, Effective WIMPs, Phys. Rev. D 89 (2014) 015011 [arXiv:1307.8120] [InSPIRE].

[7] H. An, L.-T. Wang and H. Zhang, Dark matter with t-channel mediator: a simple step beyond contact interaction, Phys. Rev. D 89 (2014) 115014 [arXiv:1308.0592] [INSPIRE].

[8] Y. Bai and J. Berger, Fermion Portal Dark Matter, JHEP 11 (2013) 171 [arXiv:1308.0612] [INSPIRE]. 
[9] A. DiFranzo, K.I. Nagao, A. Rajaraman and T.M.P. Tait, Simplified Models for Dark Matter Interacting with Quarks, JHEP 11 (2013) 014 [arXiv:1308.2679] [INSPIRE].

[10] O. Buchmueller, M.J. Dolan and C. McCabe, Beyond effective field theory for dark matter searches at the LHC, JHEP 01 (2014) 025 [arXiv:1308.6799] [inSPIRE].

[11] C. Cheung and D. Sanford, Simplified models of mixed dark matter, JCAP 02 (2014) 011 [arXiv:1311.5896] [INSPIRE].

[12] M. Papucci, A. Vichi and K.M. Zurek, Monojet versus rest of the world I: t-channel Models, arXiv: 1402.2285 [INSPIRE].

[13] A. De Simone, G.F. Giudice and A. Strumia, Benchmarks for dark matter searches at the LHC, JHEP 06 (2014) 081 [arXiv: 1402.6287] [INSPIRE].

[14] P.J. Fox and E. Poppitz, Leptophilic Dark Matter, Phys. Rev. D 79 (2009) 083528 [arXiv:0811.0399] [INSPIRE].

[15] S. Baek and P. Ko, Phenomenology of $\mathrm{U}(1)_{L_{\mu}-L_{\tau}}$ charged dark matter at PAMELA and colliders, JCAP 10 (2009) 011 [arXiv:0811.1646] [INSPIRE].

[16] P.-f. Yin, J. Liu and S.-h. Zhu, Detecting light leptophilic gauge boson at BESIII detector, Phys. Lett. B 679 (2009) 362 [arXiv:0904.4644] [INSPIRE].

[17] LUX collaboration, D.S. Akerib et al., First results from the LUX dark matter experiment at the Sanford Underground Research Facility, Phys. Rev. Lett. 112 (2014) 091303 [arXiv: 1310.8214] [INSPIRE].

[18] AMS collaboration, M. Aguilar et al., First Result from the Alpha Magnetic Spectrometer on the International Space Station: Precision Measurement of the Positron Fraction in Primary Cosmic Rays of 0.5-350 GeV, Phys. Rev. Lett. 110 (2013) 141102 [inSPIRE].

[19] B. Bertucci, Positron+electron spectrum from 0.5 gev to 700 gev, Technical Report AMS at 33rd International Cosmic Ray Conference (ICRC 2013), Rio de Janeiro Brazil (2013).

[20] V. Barger, W.-Y. Keung and G. Shaughnessy, Spin Dependence of Dark Matter Scattering, Phys. Rev. D 78 (2008) 056007 [arXiv: 0806.1962] [INSPIRE].

[21] B. Batell, T. Lin and L.-T. Wang, Flavored Dark Matter and R-Parity Violation, JHEP 01 (2014) 075 [arXiv:1309.4462] [INSPIRE].

[22] J.R. Ellis, T. Falk, K.A. Olive and M. Srednicki, Calculations of neutralino-stau coannihilation channels and the cosmologically relevant region of MSSM parameter space, Astropart. Phys. 13 (2000) 181 [Erratum ibid. 15 (2001) 413] [hep-ph/9905481] [INSPIRE].

[23] R.L. Arnowitt et al., Determining the Dark Matter Relic Density in the Minimal Supergravity Stau-Neutralino Coannihilation Region at the Large Hadron Collider, Phys. Rev. Lett. 100 (2008) 231802 [arXiv:0802.2968] [INSPIRE].

[24] J. Kopp, V. Niro, T. Schwetz and J. Zupan, DAMA/LIBRA and leptonically interacting Dark Matter, Phys. Rev. D 80 (2009) 083502 [arXiv:0907.3159] [INSPIRE].

[25] P. Agrawal, S. Blanchet, Z. Chacko and C. Kilic, Flavored Dark Matter and Its Implications for Direct Detection and Colliders, Phys. Rev. D 86 (2012) 055002 [arXiv:1109.3516] [INSPIRE].

[26] A.L. Fitzpatrick and K.M. Zurek, Dark Moments and the DAMA-CoGeNT Puzzle, Phys. Rev. D 82 (2010) 075004 [arXiv: 1007.5325] [INSPIRE]. 
[27] C.M. Ho and R.J. Scherrer, Anapole Dark Matter, Phys. Lett. B 722 (2013) 341 [arXiv: 1211.0503] [INSPIRE].

[28] E. Del Nobile, G.B. Gelmini, P. Gondolo and J.-H. Huh, Direct detection of Light Anapole and Magnetic Dipole DM, JCAP 06 (2014) 002 [arXiv:1401.4508] [INSPIRE].

[29] P. Raghavan, Table of nuclear moments, Atom. Data Nucl. Data Tabl. 42 (1989) 189.

[30] T. Banks, J.-F. Fortin and S. Thomas, Direct Detection of Dark Matter Electromagnetic Dipole Moments, arXiv:1007.5515 [INSPIRE].

[31] CTA Consortium collaboration, M. Actis et al., Design concepts for the Cherenkov Telescope Array CTA: An advanced facility for ground-based high-energy gamma-ray astronomy, Exper. Astron. 32 (2011) 193 [arXiv: 1008.3703] [INSPIRE].

[32] M. Garny, A. Ibarra, M. Pato and S. Vogl, Closing in on mass-degenerate dark matter scenarios with antiprotons and direct detection, JCAP 11 (2012) 017 [arXiv:1207.1431] [INSPIRE].

[33] M. Garny, A. Ibarra, M. Pato and S. Vogl, Internal bremsstrahlung signatures in light of direct dark matter searches, JCAP 12 (2013) 046 [arXiv:1306.6342] [INSPIRE].

[34] PAMELA collaboration, O. Adriani et al., An anomalous positron abundance in cosmic rays with energies 1.5-100 GeV, Nature 458 (2009) 607 [arXiv: 0810.4995] [INSPIRE].

[35] Fermi LAT collaboration, M. Ackermann et al., Measurement of separate cosmic-ray electron and positron spectra with the Fermi Large Area Telescope, Phys. Rev. Lett. 108 (2012) 011103 [arXiv:1109.0521] [INSPIRE].

[36] I. Cholis and D. Hooper, Dark Matter and Pulsar Origins of the Rising Cosmic Ray Positron Fraction in Light of New Data From AMS, Phys. Rev. D 88 (2013) 023013 [arXiv: 1304.1840] [INSPIRE].

[37] M. Cirelli, R. Franceschini and A. Strumia, Minimal Dark Matter predictions for galactic positrons, anti-protons, photons, Nucl. Phys. B 800 (2008) 204 [arXiv:0802.3378] [INSPIRE].

[38] Y. Bai, M. Carena and J. Lykken, The PAMELA excess from neutralino annihilation in the NMSSM, Phys. Rev. D 80 (2009) 055004 [arXiv:0905.2964] [INSPIRE].

[39] T. Sjöstrand, S. Mrenna and P.Z. Skands, A Brief Introduction to PYTHIA 8.1, Comput. Phys. Commun. 178 (2008) 852 [arXiv:0710.3820] [InSPIRE].

[40] T. Delahaye, R. Lineros, F. Donato, N. Fornengo and P. Salati, Positrons from dark matter annihilation in the galactic halo: Theoretical uncertainties, Phys. Rev. D 77 (2008) 063527 [arXiv: 0712.2312] [INSPIRE].

[41] L. Bergstrom, T. Bringmann, I. Cholis, D. Hooper and C. Weniger, New limits on dark matter annihilation from AMS cosmic ray positron data, Phys. Rev. Lett. 111 (2013) 171101 [arXiv: 1306.3983] [INSPIRE].

[42] A. Ibarra, A.S. Lamperstorfer and J. Silk, Dark matter annihilations and decays after the AMS-02 positron measurements, Phys. Rev. D 89 (2014) 063539 [arXiv:1309.2570] [INSPIRE].

[43] W. Beenakker, R. Hopker and M. Spira, PROSPINO: A Program for the production of supersymmetric particles in next-to-leading order QCD, hep-ph/9611232 [INSPIRE]. 
[44] B. Fuks, M. Klasen, D.R. Lamprea and M. Rothering, Revisiting slepton pair production at the Large Hadron Collider, JHEP 01 (2014) 168 [arXiv:1310.2621] [INSPIRE].

[45] ATLAS collaboration, Search for direct-slepton and direct-chargino production in final states with two opposite-sign leptons, missing transverse momentum and no jets in $20 / \mathrm{fb}$ of $p$ p collisions at $\sqrt{s}=8 \mathrm{TeV}$ with the ATLAS detector, ATLAS-CONF-2013-049 (2013).

[46] CMS collaboration, Search for electroweak production of charginos, neutralinos and sleptons using leptonic final states in pp collisions at 8 TeV, CMS-PAS-SUS-13-006 (2013).

[47] K.T. Matchev and M. Park, A General method for determining the masses of semi-invisibly decaying particles at hadron colliders, Phys. Rev. Lett. 107 (2011) 061801 [arXiv: 0910.1584] [INSPIRE].

[48] D.R. Tovey, On measuring the masses of pair-produced semi-invisibly decaying particles at hadron colliders, JHEP 04 (2008) 034 [arXiv:0802.2879] [INSPIRE].

[49] M.R. Buckley, J.D. Lykken, C. Rogan and M. Spiropulu, Super-Razor and Searches for Sleptons and Charginos at the LHC, Phys. Rev. D 89 (2014) 055020 [arXiv:1310.4827] [INSPIRE].

[50] C.G. Lester and D.J. Summers, Measuring masses of semiinvisibly decaying particles pair produced at hadron colliders, Phys. Lett. B 463 (1999) 99 [hep-ph/9906349] [INSPIRE].

[51] A. Barr, C. Lester and P. Stephens, m(T2): The Truth behind the glamour, J. Phys. G 29 (2003) 2343 [hep-ph/0304226] [InSPIRE].

[52] H.-C. Cheng and Z. Han, Minimal Kinematic Constraints and m(T2), JHEP 12 (2008) 063 [arXiv:0810.5178] [INSPIRE].

[53] P. Konar, K. Kong, K.T. Matchev and M. Park, Dark Matter Particle Spectroscopy at the LHC: Generalizing M(T2) to Asymmetric Event Topologies, JHEP 04 (2010) 086 [arXiv:0911.4126] [INSPIRE].

[54] Y. Kats, P. Meade, M. Reece and D. Shih, The Status of GMSB After 1/fb at the LHC, JHEP 02 (2012) 115 [arXiv:1110.6444] [INSPIRE].

[55] Y. Bai, H.-C. Cheng, J. Gallicchio and J. Gu, Stop the Top Background of the Stop Search, JHEP 07 (2012) 110 [arXiv: 1203.4813] [INSPIRE].

[56] C. Kilic and B. Tweedie, Cornering Light Stops with Dileptonic mT2, JHEP 04 (2013) 110 [arXiv:1211.6106] [INSPIRE].

[57] Y. Bai, H.-C. Cheng, J. Gallicchio and J. Gu, A toolkit of the stop search via the chargino decay, JHEP 08 (2013) 085 [arXiv: 1304.3148] [INSPIRE].

[58] UA1 collaboration, G. Arnison et al., Experimental Observation of Isolated Large Transverse Energy Electrons with Associated Missing Energy at $\sqrt{s}=540 \mathrm{GeV}$,

Phys. Lett. B 122 (1983) 103 [INSPIRE].

[59] W. van Neerven, J. Vermaseren and K. Gaemers, Lepton - jet events as a signature for $W$ production in $p$ anti-p collisions, NIKHEF-H/82-20 (1982).

[60] V.D. Barger, A.D. Martin and R.J.N. Phillips, Perpendicular $\nu_{e}$ Mass From W Decay, Z. Phys. C 21 (1983) 99 [inSPIRE].

[61] J. Smith, W.L. van Neerven and J.A.M. Vermaseren, The Transverse Mass and Width of the W Boson, Phys. Rev. Lett. 50 (1983) 1738 [INSPIRE]. 
[62] J. Alwall, M. Herquet, F. Maltoni, O. Mattelaer and T. Stelzer, MadGraph 5: Going Beyond, JHEP 06 (2011) 128 [arXiv:1106.0522] [INSPIRE].

[63] N.D. Christensen and C. Duhr, FeynRules - Feynman rules made easy, Comput. Phys. Commun. 180 (2009) 1614 [arXiv:0806.4194] [InSPIRE].

[64] J.S. Conway, Pretty Good Simulation of high-energy collisions, 090401 release.

[65] D0 collaboration, V.M. Abazov et al., Direct measurement of the $W$ boson width, Phys. Rev. Lett. 103 (2009) 231802 [arXiv:0909.4814] [INSPIRE].

[66] K. Hagiwara, R. Liao, A.D. Martin, D. Nomura and T. Teubner, $(g-2)_{\mu}$ and $\alpha\left(M_{Z}^{2}\right)$ re-evaluated using new precise data, J. Phys. G 38 (2011) 085003 [arXiv:1105.3149] [INSPIRE].

[67] Muon G-2 collaboration, G.W. Bennett et al., Final Report of the Muon E821 Anomalous Magnetic Moment Measurement at BNL, Phys. Rev. D 73 (2006) 072003 [hep-ex/0602035] [INSPIRE].

[68] B.L. Roberts, Status of the Fermilab Muon ( $g-2)$ Experiment, Chin. Phys. C 34 (2010) 741 [arXiv: 1001.2898] [INSPIRE].

[69] T. Blum et al., The Muon (g-2) Theory Value: Present and Future, arXiv:1311.2198 [INSPIRE].

[70] T. Moroi, The Muon anomalous magnetic dipole moment in the minimal supersymmetric standard model, Phys. Rev. D 53 (1996) 6565 [Erratum ibid. D 56 (1997) 4424] [hep-ph/9512396] [INSPIRE].

[71] M.S. Carena, G.F. Giudice and C.E.M. Wagner, Constraints on supersymmetric models from the muon anomalous magnetic moment, Phys. Lett. B 390 (1997) 234 [hep-ph/9610233] [INSPIRE]. 